International Mathematical Forum, Vol. 9, 2014, no. 3, 131 - 136

HIKARI Ltd, www.m-hikari.com

http://dx.doi.org/10.12988/imf.2014.312247

\title{
Portfolio Selection Problem with Stopping Time under O-U Processes
}

\author{
Yanling Wang and Limin Liu \\ College of Mathematics and Information Science \\ Henan Normal University, Xinxiang 453007, China
}

Copyright (C) 2014 Yanling Wang and Limin Liu. This is an open access article distributed under the Creative Commons Attribution License, which permits unrestricted use, distribution, and reproduction in any medium, provided the original work is properly cited.

\begin{abstract}
This paper deals with the portfolio selection problem of the exponential utility with stopping time under the O-U processes model. By using the dynamic programming and the Feynman-Kac approach, the optimal strategy and the optimal stopping time are obtained.
\end{abstract}

Keywords: O-U Processes; stopping time; HJB equation; Portfolio selection

\section{Introduction}

The portfolio selection problem is one of the important problems in modern financial mathematics. The problem was firstly proposed by Merton [1] and extended wildly later. Several researches focused on the portfolio selection problem in the past decade [2-4]. In the traditional model, we solve the problem without the time-stopping condition. However, the choice of the optimal stopping time is equally important with the optimal investment strategy, so that the study is particularly important about the portfolio selection problem with the stopping time. There are several researches about portfolio selection problem with the stopping time $[5,6]$, but they did not provide the explicit solution of the optimal investment strategy and the optimal stopping time. In this paper, we obtain the explicit solution of the portfolio selection problem with the stopping time under the exponential utility function by utilizing the method in [7]. 


\section{Market Model}

Consider a financial market that can operate the continuous trading. There are two types of assets: one is risk free assets $B_{t}$ (let $B_{t} \equiv 1$ ), and then the price process $S(t)$ of the other risk assets is the discounted price process. We suppose that the price processes of risk investments satisfy the O-U processes:

$$
d S_{t}=\alpha\left(\mu-\ln S_{t}\right) S_{t} d t+\sigma S_{t} d W_{t},
$$

where $W_{t}$ is the standard Brown motion, $\alpha, \mu, \sigma$ are constants. Then,

$$
\ln S_{t}=e^{-\alpha t} \ln S_{0}+\left(\mu-\frac{\sigma^{2}}{2 \alpha}\right)\left(1-e^{-\alpha t}\right)+\sigma \int_{0}^{t} e^{\alpha(u-t)} d W_{u} .
$$

Let the treasures of the investor is $x$ at the time $t$. When $s>t$, the shares investing on the risk assets and the risk free asset are $\pi_{s}^{0}$ and $\pi_{s}$, respectively. We have

$$
d X_{s}=\alpha\left(\mu-\ln S_{s}\right) X_{s} \pi_{s} d s+\sigma X_{s} \pi_{s} d W_{s}, t \leq s \leq T
$$

where $X_{t}=x \in R$. If $\pi$ is $\mathcal{F}_{s}$-measurable and satisfies $E \int_{t}^{T}\left\|\pi_{s}\right\|^{2} d s<\infty$, we call $\pi$ admissible, and $\mathcal{U}$ denotes the admissible strategy set. Preset the stopping time $\tau$ satisfies $0 \leq \tau \leq T$, and white $\mathcal{T}=\{\tau: \Omega \rightarrow[0, T]\}$. We use $\mathcal{A}=\{(\pi, \tau): \pi \in \mathcal{U}, \tau \in \mathcal{T}\}$ to express the control set. Consider the portfolio selection problem

$$
\left\{\begin{array}{l}
\max E\left[-e^{-\gamma\left(X_{\tau}\right)}\right] \\
\text { s.t. }(\pi, \tau) \in \mathcal{A}
\end{array}\right.
$$

of the exponential utility function with the stopping time, where $\gamma$ is the index of risk aversion.

For solving the question (3), we define

$$
V(x, s) \hat{=} \sup _{(\pi, \tau) \in \mathcal{A}} E\left[-e^{-\gamma\left(X_{\tau}\right)}\right] .
$$

Referring to [7], we have

$$
V(x, s)=\sup _{\tau \in \mathcal{T}} \sup _{\pi \in \mathcal{U}_{\tau}(x, s)} E\left[-e^{-\gamma\left(X_{\tau}\right)}\right]=\sup _{\tau \in \mathcal{T}} V^{\tau}(x, s),
$$

where $\mathcal{U}_{\tau}=\{\pi:(\pi, \tau) \in \mathcal{A}\}, V^{\tau}(x, s) \hat{=} \sup _{\pi \in \mathcal{U}_{\tau}} E\left[-e^{-\gamma\left(X_{\tau}\right)}\right]$.

For the given stopping time $\tau \in \mathcal{T}$ at random, consider the question $V^{\tau}(x, s)$. Let $t \leq s \leq T$, we define

$$
J(t, x, s ; \pi) \hat{=} E\left[-e^{-\gamma\left(X_{\tau}\right)} \mid X_{t}=x, S_{t}=s\right]
$$

and

$$
V^{\tau}(t, x, s)=\sup _{\pi \in \mathcal{U}_{\tau}} J(t, x, s ; \pi)
$$

and then $V^{\tau}(x, s)=V^{\tau}(0, x, s)$. 


\section{Optimal Investment Strategy and Optimal Stopping Time}

Theorem 3.1 The value function in the question (6) is

$$
V^{\tau}(t, x, s)=-\exp \left(-\gamma x+f_{0}(t)+f_{1}(t) \ln s+f_{2}(t)(\ln s)^{2}\right),
$$

where

$$
\begin{aligned}
& f_{0}(t)=-\frac{\alpha^{2} \mu^{2}}{2 \sigma^{2}}(\tau-t)-\frac{\alpha^{2}(\mu+1)}{4}(\tau-t)^{2}-\frac{\sigma^{2} \alpha^{2}}{24}(\tau-t)^{3}, \\
& f_{1}(t)=\frac{\alpha^{2} \mu}{\sigma^{2}}(\tau-t)+\frac{\alpha^{2}}{4}(\tau-t)^{2}, \\
& f_{2}(t)=-\frac{\alpha^{2}}{2 \sigma^{2}}(\tau-t) .
\end{aligned}
$$

The corresponding optimal investment strategy is

$$
\pi^{*}(t, s)=\frac{\alpha(\mu-\ln s)+\sigma^{2}\left(f_{1}(t)+2 f_{2}(t) \ln s\right)}{\gamma \sigma^{2}} .
$$

Proof. Utilizing the dynamical programming approach, we can obtain the value function of the question (6) by solving the HJB equation. The corresponding function of (6) is

$$
\begin{aligned}
V_{t}^{\tau}+ & \max _{\pi}\left\{\alpha(\mu-\ln s) \pi V_{x}^{\tau}+\frac{1}{2} \sigma^{2} \pi^{2} V_{x x}^{\tau}+\sigma^{2} \pi s V_{x s}^{\tau}\right\} \\
& +\alpha(\mu-\ln s) s V_{s}^{\tau}+\frac{1}{2} \sigma^{2} s^{2} V_{s s}^{\tau}=0
\end{aligned}
$$

The boundary condition is $V^{\tau}(\tau, x, s)=-e^{-\gamma x}, V^{\tau}(\tau, 0, s)=-1$.

The optimal investment strategy of the 1-order condition is

$$
\pi^{*}=-\frac{\alpha(\mu-\ln s) V_{x}^{\tau}+s \sigma^{2} V_{x s}^{\tau}}{\sigma^{2} V_{x x}^{\tau}} .
$$

Substitute the above formula to (9), we obtain

$$
V_{t}^{\tau}-\frac{1}{2} \frac{\left(\alpha(\mu-\ln s) V_{x}^{\tau}+\sigma^{2} s V_{x s}^{\tau}\right)^{2}}{\sigma^{2}+V_{x x}^{\tau}}+\alpha(\mu-\ln s) s V_{s}^{\tau}+\frac{1}{2} \sigma^{2} s^{2} V_{s s}^{\tau}=0
$$

We suppose that

$$
V^{\tau}(t, x, s)=-e^{-\gamma x+g(t, s)} .
$$

Substitute it to equation (11), we obtain that $g(t, s)$ satisfies the condition

$$
g_{t}+\frac{1}{2} \sigma^{2} s^{2} g_{s s}-\frac{\alpha^{2}(\mu-\ln s)^{2}}{2 \sigma^{2}}=0
$$

and the boundary condition is $g(\tau, s)=0$. 
For solving the equation (13), we apply the Feynman-Kac method [4]. Express $g(t, s)$ to be the expectation operator of the stochastic process $\tilde{S}_{t}$, where $d \tilde{S}_{t}=\sigma \tilde{S}_{t} d W_{t}$. Obviously,

$$
\ln \tilde{S_{u}}=\ln s+\sigma\left(W_{u}-W_{t}\right)-\frac{1}{2} \sigma^{2}(u-t),
$$

and then we have

$$
\begin{aligned}
g(t, s) & =E\left[\int_{t}^{\tau}-\frac{\alpha^{2}\left(\mu-\ln \tilde{S_{u}}\right)^{2}}{2 \sigma^{2}} d u \mid \tilde{S}_{t}=s\right] \\
& =E\left[-\frac{\alpha^{2}}{2 \sigma^{2}} \int_{t}^{\tau}\left(\mu^{2}-2 \mu \ln \tilde{S}_{u}+\left(\ln \tilde{S_{u}}\right)^{2}\right) d u \mid \tilde{S}_{t}=s\right] \\
& =-\frac{\alpha^{2} \mu^{2}}{2 \sigma^{2}}(\tau-t)+\frac{\alpha^{2} \mu}{\sigma^{2}} \\
& E\left[\int_{t}^{\tau}\left(\ln s+\sigma\left(W_{u}-W_{t}\right)-\frac{1}{2} \sigma^{2}(u-t) d u\right]\right. \\
& -\frac{\alpha^{2}}{2 \sigma^{2}} E\left[\int_{t}^{\tau}\left(\ln s+\sigma\left(W_{u}-W_{t}\right)-\frac{1}{2} \sigma^{2}(u-t)\right)^{2} d u\right] \\
& =-\frac{\alpha^{2} \mu^{2}}{2 \sigma^{2}}(\tau-t)-\frac{\alpha^{2}(\mu+1)}{4}(\tau-t)^{2}-\frac{\sigma^{2} \alpha^{2}}{24}(\tau-t)^{3} \\
& +\left(\frac{\alpha^{2} \mu}{\sigma^{2}}(\tau-t)+\frac{\alpha^{2}}{4}(\tau-t)^{2}\right) \ln s-\frac{\alpha^{2}}{2 \sigma^{2}}(\tau-t)(\ln s)^{2},
\end{aligned}
$$

by using the Feynman-Kac formula. Substitute $f_{0}(t), f_{1}(t), f_{2}(t)$ defined in theorem 1 to the above formula, we have

$$
g(t, s)=f_{0}(t)+f_{1}(t) \ln s+f_{2}(t)(\ln s)^{2} .
$$

The equation (7) is true by (12), and the equation (8) is true by substituting (7) to (10).

Applying the theorem, we have

$$
V^{\tau}(x, s)=V^{\tau}(0, x, s)=-\exp \left(-\gamma x+f_{0}(0)+f_{1}(0) \ln s+f_{2}(0)(\ln s)^{2}\right) .
$$

Since $V(x, s)=\sup _{\tau \in \mathcal{T}} V^{\tau}(x, s)$, we can obtain

$$
\begin{aligned}
& \frac{d V^{\tau}(x, s)}{d \tau}=V^{\tau}(x, s)\left[-\frac{\alpha^{2} \mu^{2}}{2 \sigma^{2}}-\frac{\alpha^{2}(\mu+1)}{2} \tau-\frac{\sigma^{2} \alpha^{2}}{8} \tau^{2}+\ln s\left(\frac{\alpha^{2} \mu}{\sigma^{2}}+\frac{\alpha^{2}}{2} \tau\right)-\frac{\alpha^{2}}{2 \sigma^{2}}(\ln s)^{2}\right] \\
& \quad=-V^{\tau}(x, s)\left[\frac{\sigma^{2} \alpha^{2}}{8} \tau^{2}+\left(\frac{\alpha^{2}(\mu+1)}{2}-\frac{\alpha^{2}}{2} \ln s\right) \tau+\left(\frac{\alpha^{2} \mu^{2}}{2 \sigma^{2}}+\frac{\alpha^{2}}{2 \sigma^{2}}(\ln s)^{2}-\ln s \frac{\alpha^{2} \mu}{\sigma^{2}}\right)\right]
\end{aligned}
$$

by computing the derivative with respect to $\tau$ of equation (14). Define

$$
f(\tau)=\frac{\sigma^{2} \alpha^{2}}{8} \tau^{2}+\left(\frac{\alpha^{2}(\mu+1)}{2}-\frac{\alpha^{2}}{2} \ln s\right) \tau+\left(\frac{\alpha^{2} \mu^{2}}{2 \sigma^{2}}+\frac{\alpha^{2}}{2 \sigma^{2}}(\ln s)^{2}-\ln s \frac{\alpha^{2} \mu}{\sigma^{2}}\right) .
$$

Since $f(\tau)$ is a quadratic function, and

$$
\Delta=\frac{\alpha^{4}}{4}(2 \mu+1-\ln s),
$$

we have: 
(1) when $2 \mu+1 \geq \operatorname{lns}$, the equation has two real roots $\tau_{1}=\frac{2}{\sigma^{2}}(\ln s-\mu-1-\sqrt{2 \mu+1-\ln s}), \tau_{2}=\frac{2}{\sigma^{2}}(\ln s-\mu-1+\sqrt{2 \mu+1-\ln s})$.

Since $\tau_{1}<0$, and $f(0)>0$, we obtain that $f(\tau)>0$ for any $\tau \geq 0$.

(2) when $2 \mu+1 \leq \ln s$, the equation has no real root. Since $f(0)>0$, we obtain that $f(\tau)>0$ for any $\tau \geq 0$.

Basing on the above analysis, we have

$$
\frac{d V^{\tau}(x, s)}{d \tau}>0
$$

for any $\tau \geq 0$. Since $0 \leq \tau \leq T$, the optimal stopping time of $\sup _{\tau \in \mathcal{T}} V^{\tau}(x, s)$ is $\tau^{*}=T$. As a result, we can write the following theorem.

Theorem 3.2 The optimal investment strategy of question (3) is

$$
\pi^{*}(t, s)=\frac{\alpha(\mu-\ln s)+\sigma^{2}\left(f_{1}(t)+2 f_{2}(t) \ln s\right)}{\gamma \sigma^{2}},
$$

where $f_{1}(t), f_{2}(t)$ are defined as theorem 1, and the optimal stopping time is

$$
\tau^{*}=T \text {. }
$$

\section{Acknowledgments}

This work is supported by Foundation and Research in Cutting-Edge Technologies in the Project of Henan Province (No.122300410396), and the Soft Science Project of Henan Province (No.122400450180).

\section{Reference}

[1] Merton.R., Optimal consumption and portfolio rules in a continuous time model[J].Journal of Economic Theory.1971,3,373-413.

[2] Benth.F.E., Karlsen.K.H., A note on merton's portflio selection problem for the Schwartz Mean-reversion model[J]. Pure Mathematics. 2004, (7):1-15

[3] Pham.H., Smooth solutions to optimal investment models with stochastic volatilities and portfolio constraints[J]. Appl Math Optim 2002,46,5578.

[4] Zariphopoulou.T., Optimal investment and consumption models with non-linear stock dynamics[J]. Math Methods Oper Res. 1999, 50(2):271296. 
[5] Karatzas. I, Zamfirescu. I., Martingale approach to stochastic control with discretionary stopping $[\mathrm{J}]$. Applied Mathematics and Optimiz ation, 2006,53:163-184.

[6] Wang Lei, A Mixed Optimal Stopping and Control Problem for Linear Stochastic Systems under Quadratic Costs[J]. Journal of Fudan University(Natural Science), 2008,47(2):195-205.

[7] Liu Limin, Xiao Qingxian, Mean-variance stochastic control for the relative return process of jump-diffusion models with Discretionary stopping $[\mathrm{J}]$. Applied Mathematics A Journal of Chinese Universities2011,26(4):390398.

Received: December 15, 2013 